TRANSACTIONS OF THE

AMERICAN MATHEMATICAL SOCIETY

Volume 354, Number 4 , Pages 1279-1298

S 0002-9947(01)02945-2

Article electronically published on November 19, 2001

\title{
GAUSSIAN BOUNDS FOR DERIVATIVES OF CENTRAL GAUSSIAN SEMIGROUPS ON COMPACT GROUPS
}

\author{
A. BENDIKOV AND L. SALOFF-COSTE
}

\begin{abstract}
For symmetric central Gaussian semigroups on compact connected groups, assuming the existence of a continuous density, we show that this density admits space derivatives of all orders in certain directions. Under some additional assumptions, we prove that these derivatives satisfy certain Gaussian bounds.
\end{abstract}

\section{INTRODUCTION}

Let $G$ be a compact connected group equipped with its normalized Haar measure $\nu$. Let $\left(\mu_{t}\right)_{t>0}$ be a weakly continuous convolution semigroup of probability measures on $G$. This means precisely that each $\mu_{t}, t>0$, is a probability measure on $G$ and that $\left(\mu_{t}\right)_{t>0}$ satisfies

(i) $\mu_{t} * \mu_{s}=\mu_{t+s}, t, s>0$

(ii) $\mu_{t} \rightarrow \delta_{e}$ weakly as $t \rightarrow 0$.

Such a semigroup is called Gaussian if it also satisfies

(iii) $t^{-1} \mu_{t}\left(V^{c}\right) \rightarrow 0$ as $t \rightarrow 0$ for any neigborhood $V$ of the identity element $e \in G$. We say that $\left(\mu_{t}\right)_{t>0}$ is symmetric if $\mu_{t}(A)=\mu_{t}\left(A^{-1}\right)$ for all $t>0$ and all Borel sets $A \subset G$. We say that $\left(\mu_{t}\right)_{t>0}$ is central if $\mu_{t}\left(a^{-1} A a\right)=\mu_{t}(A)$ for all $t>0$, all $a \in G$, and any Borel subset $A \subset G$.

Given a Gaussian semigroup $\left(\mu_{t}\right)_{t>0}$, set

$$
H_{t} f(x)=\int_{G} f(x y) d \mu_{t}(y) .
$$

The operators $\left(H_{t}\right)_{t>0}$ form a Markov semigroup. If $\mu_{t}$ is symmetric then $H_{t}$ extends to $L^{2}(G, d \nu)$ as a semigroup of self-adjoint operators. One can then associate to $\left(\mu_{t}\right)_{t>0}$ its $L^{2}(G, d \nu)$-infinitesimal generator $(-L, \operatorname{Dom}(L))$ and its Dirichlet form $(\mathcal{E}, \operatorname{Dom}(\mathcal{E}))$ so that

$$
H_{t}=e^{-t L} \quad \text { on } \quad L^{2}(G, d \nu)
$$

and

$$
\mathcal{E}(f, g)=\left\langle L^{1 / 2} f, L^{1 / 2} g\right\rangle, \quad f, g \in \operatorname{Dom}(\mathcal{E})=\operatorname{Dom}\left(L^{1 / 2}\right) .
$$

Definition 1.1. Consider a Gaussian semigroup $\left(\mu_{t}\right)_{t>0}$ on $G$.

Received by the editors May 21, 2001 and, in revised form, September 4, 2001. 2000 Mathematics Subject Classification. Primary 60B15, 43A77, 60J45, 60J60. Research partially supported by NSF Grant DMS-9802855. 
- We say that $\left(\mu_{t}\right)_{t>0}$ has property (CK) if, for all $t>0, \mu_{t}$ is absolutely continuous with respect to the Haar measure $\nu$ on $G$ and has a continuous density $x \mapsto \mu_{t}(x)$.

- We say that $\left(\mu_{t}\right)_{t>0}$ has property $(\mathrm{CK} *)$ if it has property $(\mathrm{CK})$ and that

$$
\lim _{t \rightarrow 0} t \log \mu_{t}(e)=0 .
$$

It is known (see [9, 20]) that no Gaussian semigroup can have such properties if the group $G$ is not locally connected or is not metrizable. In [5], we proved that any locally compact, connected, locally connected, metrizable group $G$ admits many symmetric Gaussian semigroups satisfying $(\mathrm{CK} *)$. In 8 , we proved that any compact, connected, locally connected, metrizable group $G$ admits a host of symmetric central Gaussian semigroups satisfying $(\mathrm{CK} *)$, and even stronger properties of this type. In [6, 7, we obtained some Gaussian estimates for the density of symmetric Gaussian semigroups satisfying $(\mathrm{CK} *)$. This Gaussian estimates differ from the classical Gaussian estimates developed by Davies and others [16, 29] in that they do not use the so-called intrinsic distance. Indeed, as explained in [6, 7], there are many symmetric Gaussian semigroups satisfying $(\mathrm{CK} *)$ for which the associated intrinsic distance is infinite almost everywhere.

The aim of the present paper is to prove Gaussian estimates for the time and space derivatives of the density $(t, x) \mapsto \mu_{t}(x)$ under the hypothesis that $\left(\mu_{t}\right)_{t>0}$ is symmetric, central, and satisfies (CK*). In order to obtain Gaussian estimates on space derivatives, we will adapt a line of reasoning introduced by the second author in [26].

Such estimates are crucial for a number of further developments concerning symmetric central Gaussian semigroups on compact groups. This is illustrated in [10, 11]. In these two papers, we show that property $(\mathrm{CK} *)$ characterizes those symmetric central Gaussian semigroups whose infinitesimal generator is hypoelliptic. Our proof that property $(\mathrm{CK} *)$ implies hypoellipticity is adapted from the line of reasoning developed in [24, Section 8] for second order differential operators in $\mathbb{R}^{n}$ (the authors are grateful to D. Stroock for asking whether hypoellipticity could be studied by the method of [24, Section 8] in the present infinite dimensional setting). This approach makes essential use of the Gaussian estimates obtained below.

\section{BACKGROUND AND NOTATION}

2.1. Projective structure. The following setup and notation will be in force throughout this article. Let $G$ be a connected compact group with neutral element $e$. Such a group contains a descending family of compact normal subgroups $K_{\alpha}$ indexed by a suitable index set $\aleph$, such that $\bigcap_{\alpha \in \aleph} K_{\alpha}=\{e\}$ and, for each $\alpha, G / K_{\alpha}$ is a Lie group. Consider the projection maps $\pi_{\alpha, \beta}: G_{\beta} \rightarrow G_{\alpha}, \beta \geq \alpha$. $G$ is the projective limit of the projective system $\left(G_{\alpha}, \pi_{\beta, \alpha}\right)_{\beta \geq \alpha}$. The Lie algebra $\mathfrak{G}$ of $G$ is then defined to be the projective limit of the Lie algebras $\mathfrak{G}_{\alpha}$ of the groups $G_{\alpha}$ equipped with the projection maps $d \pi_{\beta, \alpha}$.

Throughout the paper we assume that $G$ is compact, connected, locally connected and metrizable. The latter hypothesis is equivalent to saying that the topology of $G$ is generated by a countable basis. See [21]. Under this hypothesis, the family $K_{\alpha}, \alpha \in \aleph$, can be taken to be finite (if $G$ is a Lie group) or countable and we will assume throughout the paper that the index set $\aleph$ is indeed at most countable so that $G$ is the projective limit of the sequence of Lie groups $\left(G_{\alpha}\right)$. By results 
of Heyer and Siebert [20], the topological hypotheses that $G$ is locally connected and metrizable are necessary for the existence of Gaussian semigroups which are absolutely continuous with respect to Haar measure.

For a compact Lie group $N$, denote by $\mathcal{C}^{\infty}(N)$ the set of all smooth functions on $N$. For any compact connected group $G$, set

$$
\mathcal{B}(G)=\left\{f: G \rightarrow \mathbb{R}, f=\phi \circ \pi_{\alpha} \text { for some } \alpha \in \aleph \text { and } \phi \in \mathcal{C}^{\infty}\left(G_{\alpha}\right)\right\} .
$$

The space $\mathcal{B}(G)$ is the space of Bruhat test functions introduced in [15. We refer to [15] for a precise definition of its topology. Since $G$ is metrizable, i.e., $\aleph$ is at most countable, $\mathcal{B}(G)$ is the inductive limit of the sequence of topological vector spaces $\mathcal{C}^{\infty}\left(G_{\alpha}\right)$ ([15 p. 46]). By [15. Lemme 1], $\mathcal{B}(G)$ is independent of the choice of the family $K_{\alpha}, \alpha \in \aleph$.

By definition, a distribution on $G$ is any continuous linear functional on $\mathcal{B}(G)$. This definition was introduced in [15] and such distributions are called Bruhat distributions.

Following [13, we consider the notion of projective family and projective basis.

Definition 2.1. A family $\left(Y_{i}\right)_{i \in I}$ of $\mathfrak{G}$ is a projective family of left-invariant vector fields (w.r.t. the family $\left(K_{\alpha}\right)$ ) if it has the property that, for each $\alpha \in \aleph$, there is a finite subset $I_{\alpha} \subset I$ such that $d \pi_{\alpha}\left(Y_{i}\right)=0$ if $i \notin I_{\alpha}$. A family $\left(Y_{i}\right)_{i \in I}$ of $\mathfrak{G}$ is a projective basis of $\mathfrak{G}$ (w.r.t. the family $\left(K_{\alpha}\right)$ ) if it is projective and $\left(d \pi_{\alpha}\left(Y_{i}\right)\right)_{i \in I_{\alpha}}$ is a basis of the Lie algebra $\mathfrak{G}_{\alpha}$.

By 13, $\mathfrak{G}$ does admit a projective basis. If $\left(Y_{i}\right)_{i \in I}$ is a projective basis, we can identify $\mathfrak{G}$ with $\mathbb{R}^{I}$ as a topological vector space: For any $Z \in \mathfrak{G}$, there exists a unique $a=\left(a_{i}\right)_{i \in I}$ such that for any $\alpha \in \aleph, d \pi_{\alpha}(Z)=\sum_{i \in I} a_{i} d \pi_{\alpha}\left(Y_{i}\right)$ and convergence in $\mathfrak{G}$ is equivalent to convergence coordinate by coordinate. Since the group $G$ is assumed to be metrizable, projective families have at most a countable number of elements.

Given a projective basis $Y$, a homogeneous left-invariant differential operator of degree $k$ on $G$ is a sum

$$
P=\sum_{\ell \in I^{k}} a_{\ell} Y^{\ell}, \quad a_{\ell} \in \mathbb{C}
$$

where, for $\ell=\left(\ell_{1}, \ldots, \ell_{k}\right) \in I^{k}, Y^{\ell}=Y_{\ell_{1}} \cdots Y_{\ell_{k}}, \quad Y_{\ell_{i}} \in Y$ (this notion is in fact independent of $Y$ ). Such a $P$ can be interpreted as a linear operator from $\mathcal{B}(G)$ to $\mathcal{B}(G)$, and also as a linear operator acting on Bruhat distributions. Indeed, if $f=\phi \circ \pi_{\alpha} \in \mathcal{B}(G)$, we have

$P f(x)=\sum_{\ell \in I^{k}} a_{\ell} Y^{\ell} f(x)=\sum_{\left(\ell_{1}, \ell_{2}, \ldots, \ell_{k}\right) \in I_{\alpha}^{k}} a_{\ell}\left[d \pi_{\alpha}\left(Y_{\ell_{1}}\right) d \pi_{\alpha}\left(Y_{\ell_{2}}\right) \cdots d \pi_{\alpha}\left(Y_{\ell_{k}}\right) \phi\right]\left(\pi_{\alpha}(x)\right)$

where the sum on the right-hand side is a finite sum since $I_{\alpha}$ is finite for each $\alpha \in \aleph$.

2.2. Gaussian semigroups and sums of squares. Given a (finite or) countable set $I$, let $\mathbb{R}^{(I)}$ be the set of all $z=\left(z_{i}\right) \in \mathbb{R}^{I}$ with finitely many non-zero entries. Using [23] and the projective structure, Heyer and Born [20, 14] proved the following theorem. 
Theorem 2.2. Given a projective basis $\left(Y_{i}\right)_{i \in \mathcal{I}}$, the infinitesimal generators of symmetric Gaussian convolution semigroups on $G$ are exactly the second order leftinvariant differential operators of the form

$$
L=-\sum_{i, j \in \mathcal{I}} a_{i, j} Y_{i} Y_{j}
$$

where $A=\left(a_{i, j}\right)_{\mathcal{I} \times \mathcal{I}}$ is a real symmmetric non-negative matrix in the sense that $a_{i, j}=a_{j, i} \in \mathbb{R}$ and $\forall \xi \in \mathbb{R}^{(\mathcal{I})}, \sum a_{i, j} \xi_{i} \xi_{j} \geq 0$.

Given an infinite matrix $T=\left(t_{i, j}\right)$ indexed by a countable set $\mathcal{I}$, define

$$
j_{T}: \mathcal{I} \rightarrow \mathcal{I} \cup\{+\infty\}, \quad j_{T}(i)=\inf \left\{j: t_{i, j} \neq 0\right\}
$$

with the usual convention that $\inf \emptyset=+\infty$. Set

$$
I=\left\{i \in \mathcal{I}: j_{T}(i)<+\infty\right\} .
$$

Note that, for a given matrix $T$, the property that $j_{T}$ is increasing implies that $T$ is upper-triangular. The following lemma is simple but important. The proof is left to the reader.

Lemma 2.3. Let $A=\left(a_{i, j}\right)$ be an infinite symmetric non-negative matrix indexed by a countable set $\mathcal{I}$. There exists an infinite matrix $T=\left(t_{i, j}\right)_{\mathcal{I} \times \mathcal{I}}$ such that $j_{T}$ is increasing and

$$
\forall \xi \in \mathbb{R}^{(\mathcal{I})}, \quad \sum_{i, j \in \mathcal{I}} a_{i, j} \xi_{i} \xi_{j}=\sum_{k \in I} \eta_{k}^{2}
$$

where $I$ is given by (2.2) and

$$
\forall i \in I, \quad \eta_{i}=\sum_{j \in \mathcal{I}} t_{i, j} \xi_{j} .
$$

In other words, $A=T^{t} T$. The matrix $A$ is positive, i.e.,

$$
\forall \xi \in \mathbb{R}^{(\mathcal{I})} \backslash\{0\}, \quad \sum_{i, j} a_{i, j} \xi \xi_{j}>0,
$$

if and only if $t_{i, i}>0$ for all $i \in \mathcal{I}$.

Theorem 2.4. Fix a projective basis $Y=\left(Y_{i}\right)_{\mathcal{I}}$ and $L=-\sum_{i, j \in \mathcal{I}} a_{i, j} Y_{i} Y_{j}$ with $A$ symmetric non-negative. Let I be defined by (2.2). Let $T=\left(t_{i, j}\right)_{\mathcal{I} \times \mathcal{I}}$ be the matrix given by Lemma 2.3. The family $X=\left(X_{i}\right)_{I}$ given by

$$
X_{i}=\sum_{j} t_{i, j} Y_{j}, \quad i \in I,
$$

is a projective family of linearly independent vectors and yields a decomposition of $-L$ as a sum of squares:

$$
\forall f \in \mathcal{B}(G), \quad L f=-\sum_{I} X_{i}^{2} f .
$$

Proof. The fact that $T$ is upper-triangular and $Y$ is a projective basis implies that $X$ is a projective family. Hence, for any $f \in \mathcal{B}(G)$, the sum $\sum_{I} X_{i}^{2} f$ reduces to a finite sum. Plugging the definition of the $X_{i}$ 's in terms of the $Y_{i}^{\prime}$ 's in $\sum_{I} X_{i}^{2} f$ shows that this sum equals $-L f$.

Note that the family $X$ of Theorem 2.4 is a projective basis if and only if $t_{i, i}>0$ for all $i \in \mathcal{I}$. In this case $\mathcal{I}=I$. Note also that, for a given $L$, there are many decompositions of $L$ as minus a sum of squares. 
2.3. The Hilbert space of good directions. Define the field operator $\Gamma$ to be the symmetric bilinear form

$$
\Gamma(f, g)=\frac{1}{2}(-L(f g)+f L g+g L f)
$$

on the space $\mathcal{B}(G)$ of Bruhat test functions. Computing $\Gamma$ in a projective family (not necessarily a basis) $X=\left(X_{i}\right)_{i \in I}$ where $L=-\sum_{i \in I} X_{i}^{2}$ we find

$$
\Gamma(f, g)=\sum_{i \in I}\left(X_{i} f\right)\left(X_{i} g\right) .
$$

The next definition plays a crucial role in this paper.

Definition 2.5. Given the generator $-L$ of a symmetric Gaussian semigroup on $G$, let $\mathcal{H}(L)$ be the vector space

$$
\mathcal{H}(L)=\left\{Z \in \mathfrak{G}: \exists c(Z), \forall f \in \mathcal{B}(G),|Z f(e)|^{2} \leq c(Z) \Gamma(f, f)(e)\right\}
$$

equipped with the norm

$$
\|Z\|_{L}=\sup _{\substack{f \in \mathcal{B}(G) \\ \Gamma(f, f)(e) \leq 1}}\{|Z f(e)|\}
$$

We now give a different description of $\mathcal{H}(L)$.

Lemma 2.6. The space $\mathcal{H}(L)$ equipped with the norm $\|Z\|_{L}$ is a Hilbert space. In particular, for any projective family $X=\left(X_{i}\right)_{i \in I}$ of linearly independent vectors such that $L=-\sum_{i \in I} X_{i}^{2}$, we have

$$
\mathcal{H}(L)=\left\{Z=\sum_{i \in I} \zeta_{i} X_{i}: \sum_{i \in I}\left|\zeta_{i}\right|^{2}<\infty\right\}
$$

and, for all $Z=\sum_{i \in I} \zeta_{i} X_{i}$,

$$
\|Z\|_{L}^{2}=\sum_{i \in I}\left|\zeta_{i}\right|^{2}
$$

Proof. For $L=-\sum_{i \in I} X_{i}^{2}, \Gamma$ is given by (2.4). Thus, if $Z=\sum_{i \in I} \zeta_{i} X_{i}$ with $\sum\left|\zeta_{i}\right|^{2}<\infty, Z \in \mathcal{H}(L)$, and

$$
\|Z\|_{L}^{2} \leq\left(\sum_{i \in I}\left|\zeta_{i}\right|^{2}\right)
$$

Let us first assume that $X=\left(X_{i}\right)_{I}$ is a projective family extracted from a projective basis $\left(X_{i}\right)_{\mathcal{I}}$. Let $Z=\sum_{\mathcal{I}} \zeta_{i} X_{i}$ be an arbitrary left-invariant vector field. For any finite subset $J \subset \mathcal{I}$ and any sequence $\xi=\left(\xi_{j}\right)_{J}$, we can find $f_{J}^{\xi} \in \mathcal{B}(G)$ such $X_{j} f_{J}^{\xi}(e)=\xi_{j}$ if $j \in J, X_{j} f_{J}^{\xi}(e)=0$ if $j \notin J$. Then we have

$$
\left|Z f_{J}^{\xi}(e)\right|^{2}=\left(\sum_{j \in J} \zeta_{j} \xi_{j}\right)^{2} \text { and } \Gamma\left(f_{J}^{\xi}, f_{J}^{\xi}\right)=\sum_{j \in J \cap I}\left|\xi_{j}\right|^{2} .
$$

Thus if $Z \in \mathcal{H}(L)$ then we must have $\zeta_{i}=0$ for all $i \in \mathcal{I} \backslash I$ and also $\sum_{I}\left|\zeta_{i}\right|^{2}<\infty$. Moreover,

$$
\|Z\|_{L}^{2} \geq \sup _{\xi} \frac{\left|Z f_{J}^{\xi}(e)\right|^{2}}{\Gamma\left(f_{J}^{\xi}, f_{J}^{\xi}\right)}=\sum_{j \in J}\left|\zeta_{j}\right|^{2} .
$$


Since this holds for any finite subset $J \subset I$, we conclude that

$$
\|Z\|_{L}^{2} \geq \sum_{i \in I}\left|\zeta_{i}\right|^{2}
$$

as desired. A simple Hilbert space argument then shows that any independent projective family $\left(X_{i}\right)_{I}$ such that $L=-\sum X_{i}^{2}$ must be a basis of $\mathcal{H}(L)$.

Remark 2.7. The space $\mathcal{H}(L)$ must be interpreted as a space of good directions in $\mathfrak{G}$. It captures very important non-trivial information about $L$ and necessarily plays a crucial role in any precise analysis of $L$ and the associated Gaussian semigroup. For instance, the one parameter subgroups associated to directions in $\mathcal{H}(L)$ are rectifiable for the intrinsic distance. See, e.g., 6] 7] and Definition 4.7.

Example 2.8. Let $G=\mathbf{T}=\mathbf{R} / 2 \pi \mathbf{Z}$ where $\mathbf{R}=\mathbb{R}^{\infty}$ and $\mathbf{Z}=\mathbb{Z}^{\infty}$. Thus, $\mathbf{T}$ is the countable product of circle groups, each isomorphic to $\mathbb{T}=\mathbb{R} / 2 \pi \mathbb{Z}$. However, for the following discussion it is important to observe that $\mathbf{T}$ is defined independently of the product structure. Writing $\mathbf{T}$ as an infinite product yields a projective basis of its Lie algebra $\mathbf{R}=\mathbb{R}^{\infty}$, say $Y=\left(Y_{i}\right)_{1}^{\infty}$, where $Y_{i}=\partial_{i}$ can be identified with partial differentiation in the $i$-th coordinate. Any symmetric Gaussian semigroup $\left(\mu_{t}\right)_{t>0}$ is determined by a matrix $A=\left(a_{i, j}\right)$ as explained above. One usually says that $\left(\mu_{t}\right)_{t>0}$ is diagonal if $A$ is a diagonal matrix with $a_{i, i}=a_{i}$ and quite a lot is known about the properties of $\left(\mu_{t}\right)_{t>0}$ in this case. See [2, 3, 6, 12, In such a case, $\mathcal{H}(L)$ is the Hilbert space contained in $\mathbf{R}$ with orthonormal Hilbert basis

$$
\left(a_{i}^{1 / 2} \partial_{i}\right)_{i \in I}, \quad I=\left\{i: a_{i}>0\right\} .
$$

Let us now look at two non-diagonal $A$ 's:

$$
A_{1}=\left(\begin{array}{cccccccc}
1 & 1 & 1 & 1 & 1 & 1 & . & . \\
1 & 2 & 2 & 2 & 2 & 2 & . & . \\
1 & 2 & 3 & 3 & 3 & 3 & . & . \\
1 & 2 & 3 & 4 & 4 & 4 & . & . \\
1 & 2 & 3 & 4 & 5 & 5 & . & . \\
1 & 2 & 3 & 4 & 5 & 6 & . & . \\
. & . & . & . & . & . & . & . \\
. & . & . & . & . & . & . & .
\end{array}\right), A_{2}=\left(\begin{array}{cccccccc}
1 & \frac{1}{2} & 0 & 0 & 0 & 0 & . & . \\
\frac{1}{2} & \frac{5}{4} & \frac{2}{3} & 0 & 0 & 0 & . & . \\
0 & \frac{2}{3} & \frac{13}{9} & \frac{3}{4} & 0 & 0 & . & . \\
0 & 0 & \frac{3}{4} & \frac{25}{16} & \frac{4}{5} & 0 & . & . \\
0 & 0 & 0 & \frac{4}{5} & \frac{41}{25} & \frac{5}{6} & . & . \\
0 & 0 & 0 & 0 & \frac{5}{6} & \frac{61}{36} & . & . \\
. & . & . & . & . & . & . & . \\
. & . & . & . & . & . & . & .
\end{array}\right) .
$$

Thus, for $A_{1}, a_{i, j}=\min \{i, j\}$ whereas, for $A_{2}, a_{i, i}=1+\left(\frac{i-1}{i}\right)^{2}, a_{i, i+1}=\frac{i}{i+1}$, $a_{i-1, i}=\frac{i-1}{i}$ and $a_{i, j}=0$ if $|i-j| \geq 2$. A simple calculation shows that the corresponding Hilbert space $\mathcal{H}(L)$ has orthonormal basis $X=\left(X_{i}\right)_{1}^{\infty}$ given by

$$
X_{i}=\left\{\begin{array}{cc}
\sum_{j \geq i} Y_{i} & \text { for } A_{1}, \\
Y_{i}+\frac{i}{i+1} Y_{i+1} & \text { for } A_{2} .
\end{array}\right.
$$

In both cases, the family $\left(X_{i}\right)_{1}^{\infty}$ is also a projective basis of the Lie algebra $\mathbf{R}$ of $\mathbf{T}$.

The case of $A_{1}$ : Consider the "integer lattice"

$$
\mathbf{Z}_{X}=\left\{Z=\sum_{1}^{\infty} z_{i} X_{i}: z_{i} \in \mathbb{Z}\right\} \subset \mathbf{R}
$$

and observe that it coincides in the case of $A_{1}$ with the original integer lattice $\mathbf{Z}=\mathbf{Z}_{Y}$. Since the infinitesimal generator of $\left(\mu_{t}\right)_{t>0}$ is $\sum X_{i}^{2}$, this means that 
the Gaussian semigroup $\left(\mu_{t}\right)_{t>0}$ associated to $A_{1}$ is exactly the infinite product of identical standard Gaussian semigroups on the circles

$$
\left[\mathbb{R} X_{i}\right] /\left[2 \pi \mathbb{Z} X_{i}\right] .
$$

To illustrate what this says, observe that Kakutani's theorem implies easily that the measure $\mu_{t}$ is singular with respect to Haar measure for each $t>0$.

The case of $A_{2}$ : In this case, we cannot find a basis which "diagonalizes" $\left(\mu_{t}\right)_{t>0}$. One can ask what is a "good" basis to study $\left(\mu_{t}\right)_{t>0}$ but it seems hard to make this precise. For instance, one may want to try $X_{i}^{\prime}=Y_{i}+Y_{i+1}$ since $X_{i}$ tends to $X_{i}^{\prime}$ as $i$ tends to infinity and the integer lattice $\mathbf{Z}_{X^{\prime}}$ coincides with the original one. But, in $X^{\prime}=\left(X_{i}^{\prime}\right)_{1}^{\infty}$, the matrix $A^{\prime}$ representing $\left(\mu_{t}\right)_{t>0}$ has

$$
a_{i, i}^{\prime}=1+\sum_{2}^{i} \frac{1}{j^{2}} \text { and } a_{i, j}^{\prime}=(-1)^{j-i}\left(\frac{1}{i+1}+\sum_{2}^{i} \frac{1}{j^{2}}\right) \text { for } j>i,
$$

which is not easy at all to interpret. Developing a theory to study this kind of examples appears to be a real challenge. For instance, although we strongly suspect that the present Gaussian semigroup $\left(\mu_{t}\right)_{t>0}$ is singular with respect to Haar measure for all $t>0$, we have no proof of this fact at the present writing.

\section{Spaces of SMooth FunCtions}

3.1. The spaces $\mathcal{C}_{X}^{k}$. Any left-invariant vector field $Z \in \mathfrak{G}$ generates a one parameter group $t \mapsto e^{t Z}$ in $G$. By definition, a function $f: G \mapsto \mathbb{R}$ has a derivative at $x$ in the direction of $Z$ if

$$
Z f(x)=\lim _{t \rightarrow 0} \frac{f\left(x e^{t Z}\right)-f(x)}{t}=\left.\frac{\partial}{\partial t} f\left(x e^{t Z}\right)\right|_{t=0}
$$

exists. For $Z_{i} \in \mathfrak{G}, Z_{1} \cdots Z_{k} f(x)=Z_{1}\left[Z_{2} \cdots Z_{k} f\right](x)$ is defined inductively and we set

$$
D_{x}^{k} f\left(Z_{1}, \ldots, Z_{k}\right)=Z_{1} \cdots Z_{k} f(x) .
$$

For instance, for all $x \in G$, any function $f$ in $\mathcal{B}(G)$ has a derivative at $x$ in any direction $Z \in \mathfrak{G}$ and $D_{x}^{k} f$ is a $k$-linear form on $\mathfrak{G}$.

The proof of the following classical statement is left to the reader.

Lemma 3.1. Let $u$ be a continuous function and $Z \in \mathfrak{G}$. Consider $u$ as a Bruhat distribution and assume that the Bruhat distribution $Z u$ can be represented by a continuous function $v$. Then $u$ has a continuous derivative $Z u$ in the direction of $Z$ and $Z u=v$.

Fix a projective family $X=\left(X_{i}\right)_{i \in I}$ of $\mathfrak{G}$. Let $\mathbb{N}=\{0,1,2, \ldots\}$. For any $k \in \mathbb{N}$ and any $\ell \in I^{k}$, consider the seminorms on $\mathcal{B}(G)$ defined by

$$
N_{X}^{\ell}(f)=\left\|X^{\ell} f\right\|_{\infty}=\sup _{G}\left|X^{\ell} f\right|, \quad \ell \in I^{k} .
$$

Definition 3.2. Let $\mathcal{C}^{0}(G)=\mathcal{C}(G)$ be the set of all continuous functions on $G$ and, for each $k=1,2, \ldots$, let $\mathcal{C}_{X}^{k}$ be the linear space of all continuous functions $f: G \rightarrow \mathbb{R}$ such that, for each $\alpha \leq k$ and each $i \in I^{\alpha}, X^{i} f=X_{i_{1}} \ldots X_{i_{\alpha}} f$ exists and is a continuous function on $G$. The space $\mathcal{C}_{X}^{k}$ is equipped with the topology defined by the family of seminorms $N_{X}^{\ell}, \ell \in I^{m}, m=0,1, \ldots, k$. Set also

$$
\mathcal{C}_{X}^{\infty}=\bigcap_{k \in \mathbb{N}} \mathcal{C}_{X}^{k}
$$


equipped with the seminorms $N_{X}^{\ell}, \ell \in I^{k}, k=0,1,2, \ldots$

Recall that the left and right convolutions of a function $f \in \mathcal{C}(G)$ and a measure $\mu$ are defined by

$$
\mu * f(x)=\int_{G} f\left(y^{-1} x\right) d \mu(y), \quad f * \mu(x)=\int_{G} f\left(x y^{-1}\right) d \mu(y) .
$$

With this notation, the semigroup of operators $\left(H_{t}\right)_{t>0}$ associated to a Gaussian convolution semigroup $\left(\mu_{t}\right)_{t>0}$ on $G$ is given by $H_{t} f=f * \check{\mu}_{t}$ where $\check{\mu}(B)=\mu\left(B^{-1}\right)$ for any Borel set $B$ and any Borel measure $\mu$. If $\mu$ is central, i.e., $\mu\left(a^{-1} B a\right)=\mu(B)$ for any Borel set $B$ and any $a \in G$, then $f * \mu=\mu * f$. Thus, for any symmetric central Gaussian semigroup $\left(\mu_{t}\right)_{t>0}, H_{t} f=f * \mu_{t}=\mu_{t} * f$. [10].

The following proposition gathers some properties of the spaces $\mathcal{C}_{X}^{k}$. See, e.g.,

Lemma 3.3. Fix a projective family $X$ and $k=0,1,2, \ldots$

1. For any Borel measure $\mu$ of total mass $\|\mu\|$,

$$
\forall f \in \mathcal{C}_{X}^{k}, \quad\left\|X^{\ell}(\mu * f)\right\|_{\infty} \leq\|\mu\|\left\|X^{\ell} f\right\|_{\infty} .
$$

2. Let $\phi_{n} \in L^{1}(G), \phi_{n} \rightarrow \delta_{e}$. Then, for any $f \in \mathcal{C}_{X}^{k}, f_{n}=\phi_{n} * f$ converges to $f$ in $\mathcal{C}_{X}^{k}$.

3. $\mathcal{B}(G)$ is dense in $\mathcal{C}_{X}^{k}$.

4. $\mathcal{C}_{X}^{k}$ is an algebra for pointwise multiplication.

5. Let $E \subset \mathcal{C}(G)$ be such that, for any projective basis $Y, E \subset \mathcal{C}_{Y}^{\infty}$. Then $E \subset \mathcal{B}(G)$.

3.2. The spaces $\mathcal{S}_{X}^{k}$. Fix a projective family $X=\left(X_{i}\right)$. For $f \in \mathcal{B}(G)$, set

$$
\left|D_{x}^{k} f\right|_{X}=\left(\sum_{\left(\ell_{1}, \ell_{2}, \ldots, \ell_{k}\right) \in I^{k}}\left|D_{x}^{k} f\left(X_{\ell_{1}}, X_{\ell_{2}}, \ldots, X_{\ell_{k}}\right)\right|^{2}\right)^{1 / 2} .
$$

Consider also the function $\left|D^{k} f\right|_{X}: G \rightarrow[0,+\infty]$ defined by

$$
x \mapsto\left|D^{k} f\right|_{X}(x)=\left|D_{x}^{k} f\right|_{X}
$$

and set

$$
\left\|\left|D^{m} f\right|_{X}\right\|_{\infty}=\sup _{x \in G}\left\{\left|D_{x}^{m} f\right|_{X}\right\}, \quad S_{X}^{k}(f)=\sup _{m \leq k}\left\|\left|D^{m} f\right|_{X}\right\|_{\infty} .
$$

Definition 3.4. Given a projective family $X=\left(X_{i}\right)$, let $\mathcal{S}_{X}^{k}$ be the closure of $\mathcal{B}(G)$ for the norm $S_{X}^{k}(f)$. Let $\mathcal{S}_{X}^{\infty}$ be the space

$$
\mathcal{S}_{X}^{\infty}=\bigcap_{k \in \mathbb{N}} \mathcal{S}_{X}^{k}
$$

equipped with the topology defined by the family of seminorms $S_{X}^{k}, k=0,1,2, \ldots$.

The spaces $\mathcal{S}_{X}^{k}$ have the followig simple but remarkable property.

Proposition 3.5. Let $X=\left(X_{i}\right)_{I}$ and $Z=\left(Z_{j}\right)_{J}$ be two projective families such that $\sum_{I} X_{i}^{2}=\sum_{J} Z_{j}^{2}$ on $\mathcal{B}(G)$. Then, for each $k$,

$$
\forall f \in \mathcal{B}(G), \quad\left|D^{k} f\right|_{X}=\left|D^{k} f\right|_{Z}
$$

In particular, $\mathcal{S}_{X}^{k}=\mathcal{S}_{Z}^{k}$. 
Proof. It suffices to show that for any $f \in \mathcal{B}(G)$,

$$
\left|D_{e}^{k} f\right|_{X}^{2}=\left|D_{e}^{k} f\right|_{Z}^{2}
$$

We can assume without loss of generality that these two families $X, Z$, are indexed by the same countable set $I$. We can also assume that $X$ is extracted from a projective basis $\left(X_{i}\right)_{i \in \mathcal{I}}$. Set $L=-\sum_{I} X_{i}^{2}=-\sum_{I} Z_{i}^{2}$. By (2.4), we have

$$
\forall f, g \in \mathcal{B}(G), \quad \Gamma(f, g)=\sum_{I} X_{i} f X_{i} g=\sum_{I} Z_{i} f Z_{i} g .
$$

Moreover, each $X_{i}, Z_{i}$ belongs to $\mathcal{H}(L)$ and, by Lemma 2.6 $X$ is a basis of the Hilbert space $\mathcal{H}(L)$. Thus there are coefficients $b_{i, j}$ such that

$$
\forall i \in I, \quad Z_{i}=\sum_{j \in I} b_{i, j} X_{j} .
$$

As in the proof of Lemma 2.6, observe that for any sequence $\xi=\left(\xi_{i}\right)$ with finitely many non-zero entries we can find a function $f \in \mathcal{B}(G)$ such that $X_{i} f(e)=\xi_{i}$ (here we use the independence of the family $X$ ). Thus, (3.3) yields

$$
\forall \xi, \zeta \in \mathbb{R}^{(I)}, \quad \sum_{I} \xi_{i} \zeta_{i}=\sum_{i} \sum_{n, m} b_{i, n} b_{i, m} \xi_{n} \zeta_{m} .
$$

That is

$$
\forall n, m \in I, \quad \sum_{i} b_{i, n} b_{i, m}=\delta_{n, m}
$$

where $\delta_{n, m}=1$ if $n=m$ and 0 otherwise.

Now, write

$$
\begin{aligned}
\sum_{\ell \in I^{k}}\left|Z^{\ell} f\right|^{2} & =\sum_{\ell \in I^{k}} \sum_{n, m \in I^{k}} b_{\ell_{1}, n_{1}} \cdots b_{\ell_{k}, n_{k}} b_{\ell_{1}, m_{1}} \cdots b_{\ell_{k}, m_{k}} X^{n} f X^{m} f \\
& =\sum_{n \in I^{k}}\left|X^{n} f\right|^{2}
\end{aligned}
$$

where the last equality uses (3.4) for each $\left(n_{j}, m_{j}\right) \in I \times I$.

The following proposition gathers some important properties of the spaces $\mathcal{S}_{X}^{k}$.

Proposition 3.6. Fix a projective family $X=\left(X_{i}\right)_{I}$.

(1) Let $\mu$ be a Borel measure of total mass $\|\mu\|=|\mu|(G)$. Then

$$
\forall f \in \mathcal{S}_{X}^{k}, \quad S_{X}^{k}(\mu * f) \leq\|\mu\| S_{X}^{k}(f) .
$$

(2) A function $f$ is in $\mathcal{S}_{L}^{k}$ if and only if, for any $\ell \leq k$ and $j \in I^{\ell}$, the functions $x \mapsto X^{j} f(x)$, and $x \mapsto\left|D_{x}^{\ell} f\right|_{L}$ exists and are continuous on $G$.

(3) Let $\phi_{n} \in L^{1}(G), \phi_{n} \rightarrow \delta_{e}$ as $n$ tends to infinity. Then, for any function $f \in \mathcal{S}_{L}^{k}$, the sequence $f_{n}=\phi_{n} * f$ converges to $f$ in $\mathcal{S}_{L}^{k}$.

(4) The spaces $\mathcal{S}_{L}^{k}$ are algebras for pointwise multiplication and, for any $f, g \in \mathcal{S}_{L}^{k}$,

$$
\left|D^{k}(f g)\right|_{X} \leq 4^{k} \sup _{n \leq k}\left\{\left|D^{n} f\right|_{X}\right\} \sup _{n \leq k}\left\{\left|D^{n} g\right|_{X}\right\} .
$$

Proof of (1). For any $f \in \mathcal{B}(G)$ and $\ell \in I^{m}$,

$$
X^{\ell}(\mu * f)(x)=\int_{G} X^{\ell} f\left(y^{-1} x\right) d \mu(y) .
$$


Minkowski's inequality and (3.5) yield

$$
\left|D_{x}^{m}(\mu * f)\right|_{X} \leq \int_{G}\left|D_{y^{-1} x}^{m} f\right|_{X} d|\mu|(y) \leq\|\mu\|\left\|\left.|| D^{m} f\right|_{X}\right\|_{\infty}
$$

for any integers $m$. The desired conclusion follows.

Proof of (2) and (3). Assume that $f \in \mathcal{S}_{X}^{k}$. Then, for each $m \leq k$ and each $\ell \in I^{m}$, the function $X^{\ell} f$ is continuous as the uniform limit of continuous functions. The function

$$
\left|D_{x}^{m} f\right|_{X}: x \mapsto\left|D_{x}^{m} f\right|_{X}
$$

is also continuous as the uniform limit of continuous functions. Indeed, if $f_{n} \rightarrow f$ in $\mathcal{S}_{X}^{k}$ and $f_{n} \in \mathcal{B}(G), x \mapsto\left|D_{x}^{m} f_{n}\right|_{X}$ is a continuous function since it is, in fact, a finite sum of continuous functions.

Keeping the same notation, assume now that $f, X^{\ell} f,\left|D^{m} f\right|_{X}$ are continuous functions. Let $\phi_{n} \in \mathcal{B}(G), \phi_{n} \rightarrow \delta_{e}$ and set $f_{n}=\phi_{n} * f \in \mathcal{B}(G)$. Note that

$$
K=\sup _{n} \int_{G}\left|\phi_{n}\right| d \nu<+\infty .
$$

By a classical argument $X^{\ell} f_{n}=\phi_{n} *\left[X^{\ell} f\right]$ tends to $X^{\ell} f(x)$, uniformly in $G$. As $x \mapsto$ $\left|D_{x}^{m} f\right|_{X}$ is continuous, Dini's theorem shows that the partial sums $\sum_{\ell \in J}\left|X^{\ell} f(x)\right|^{2}$ converge uniformly to $\left|D_{x}^{m} f\right|_{X}^{2}$ as the finite set $J \subset I^{m}$ increases to $I^{m}$. Hence, for any $\epsilon>0$ there exists a finite set $J$ such that

$$
\forall x \in G, \quad \sum_{\ell \in J^{c}}\left|X^{\ell} f(x)\right|^{2} \leq \epsilon .
$$

As

$$
\sup _{x \in G}\left(\sum_{j \in J^{c}}\left|X^{\ell} f_{n}(x)\right|^{2}\right)^{1 / 2} \leq K \sup _{x \in G}\left(\sum_{j \in J^{c}}\left|X^{\ell} f(x)\right|^{2}\right)^{1 / 2},
$$

we obtain

$$
\left|D_{x}^{m}\left(f_{n}-f\right)\right|_{X}^{2} \leq \sum_{j \in J}\left|X^{\ell}\left(f_{n}-f\right)(x)\right|^{2}+(1+K) \epsilon .
$$

This shows that $S_{X}^{k}\left(f_{n}-f\right) \rightarrow 0$. Hence, $f$ belongs to $\mathcal{S}_{X}^{k}$ as desired. The same line of reasoning proves (3).

Proof of (4). For $f, g \in \mathcal{B}(G)$, and $i \in I^{m}, m \leq k$, write

$$
X^{i}(f g)=\sum_{\epsilon \in\{0,1\}^{m}} X^{\epsilon, i} f X^{\epsilon^{\prime}, i} g
$$

where $\epsilon^{\prime}$ is the "complement" of $\epsilon$ obtained by adding 1 modulo 2 to each coordinate and

$$
X^{\epsilon, i}=X_{i_{1}}^{\epsilon_{1}} \cdots X_{i_{m}}^{\epsilon_{m}}, \quad X_{j}^{1} h=X_{i} h, \quad X_{j}^{0} h=h .
$$


Thus, setting $|\epsilon|=\sum_{1}^{m} \epsilon_{i}$,

$$
\begin{aligned}
\sum_{i \in I^{m}}\left|X^{i}(f g)\right|^{2} & \leq 2^{m} \sum_{\epsilon \in\{0,1\}^{m}} \sum_{i \in I^{m}}\left|X^{\epsilon, i} f\right|^{2}\left|X^{\epsilon^{\prime}, i} g\right|^{2} \\
& =2^{m} \sum_{\epsilon \in\{0,1\}^{m}}\left(\sum_{j \in I^{|\epsilon|}}\left|X^{j} f\right|^{2}\right)\left(\sum_{j \in I^{\left|\epsilon^{\prime}\right|}}\left|X^{j} g\right|^{2}\right) \\
& \leq 4^{m} \sup _{n \leq m}\left\{\left|D^{n} f\right|_{X}^{2}\right\} \sup _{n \leq m}\left\{\left|D^{n} g\right|_{X}^{2}\right\} .
\end{aligned}
$$

Now, if $f_{n}, g_{n} \in \mathcal{B}(G)$ and $f_{n} \rightarrow f, g_{n} \rightarrow g$ in $\mathcal{S}_{X}^{k}$, it easily follows from the inequality above that $S_{X}^{k}\left(f_{n} g_{n}-f g\right) \rightarrow 0$. This proves (4).

3.3. The spaces $\mathcal{T}_{L}^{k}$ associated with bi-invariant $L$. Now let $L$ be the infinitesimal generator of a symmetric central Gaussian semigroup $\left(\mu_{t}\right)_{t>0}$. The hypothesis that $\left(\mu_{t}\right)_{t>0}$ is central is equivalent to the fact that $L$ is bi-invariant. This section introduces some spaces of smooth functions precisely adapted to $L$. Let $X=\left(X_{i}\right)_{i \in I}$ be a projective family such that (such a family always exists by Lemma 2.4)

$$
L=-\sum_{i \in I} X_{i}^{2}
$$

By Proposition 3.5 we can denote the spaces $\mathcal{S}_{X}^{k}$ by $\mathcal{S}_{L}^{k}$ since they depend only on $L$. In fact, when $L$ is bi-invariant, one can describe $\mathcal{S}_{L}^{k}$ intrinsically as follows. Recall that the iterated gradient $\Gamma_{n}$ is defined recursively for $n=1,2,3, \ldots$, by

$$
\Gamma_{n}(f, g)=\frac{1}{2}\left(-L \Gamma_{n-1}(f, g)+\Gamma_{n-1}(f, L g)+\Gamma_{n-1}(L f, g)\right)
$$

with $\Gamma_{0}(f, g)=f g$. See [1] 25] and the references therein. Higher iterated gradients, are difficult to compute in general but, since $L$ is bi-invariant, we have

$$
\forall f, g \in \mathcal{B}(G), \quad \Gamma_{n}(f, g)=\sum_{\left(\ell_{1}, \ldots, \ell_{n}\right) \in I^{n}}\left(X_{\ell_{1}} \cdots X_{\ell_{n}} f\right)\left(X_{\ell_{1}} \cdots X_{\ell_{n}} g\right) .
$$

In particular $\left|D^{n} f\right|_{X}^{2}=\Gamma_{n}(f, f)$ and we set

$$
\left|D^{n} f\right|_{L}^{2}=\left|D^{n} f\right|_{X}^{2}=\Gamma_{n}(f, f) .
$$

Now, for two integers $n, m$, define $w(n, m)=n+2 m$ and set, for any $f \in \mathcal{B}(G)$,

$$
M_{L}^{k}(f)=\sup _{x \in G} \sup _{\substack{(n, m) \in \mathbb{N}^{2} \\ w(n, m) \leq k}}\left\{\left|D_{x}^{n} L^{m} f\right|_{L}\right\} .
$$

Definition 3.7. Given a symmetric central Gaussian semigroup $\left(\mu_{t}\right)_{t>0}$ on $G$ with infinitesimal generator $-L$, let $\mathcal{T}_{L}^{k}$ be the closure of $\mathcal{B}(G)$ for the norm $M_{L}^{k}(f)$. Define $\mathcal{T}_{L}=\mathcal{T}_{L}^{\infty}$ to be the space

$$
\mathcal{T}_{L}^{\infty}=\bigcap_{k \in \mathbb{N}} \mathcal{T}_{L}^{k}
$$

equipped with the topology defined by the family of seminorms $M_{L}^{k}, k=0,1,2, \ldots$.

Note that, if $X=\left(X_{i}\right)$ is a projective family such that $L=-\sum X_{i}^{2}$, we have

$$
\mathcal{B}(G) \subset \mathcal{T}_{L}^{k} \subset \mathcal{S}_{L}^{k} \subset \mathcal{C}_{X}^{k} \subset \mathcal{C}(G) .
$$

Proposition 3.6 has an exact analog concerning the spaces $\mathcal{T}_{L}^{k}$. For instance, these spaces are algebras for pointwise multiplication. See [11]. For the purpose of the 
present paper, we only need to record the following alternative description of $\mathcal{T}_{L}^{k}$. The proof is entirely similar to that of Proposition 3.6)(2).

Proposition 3.8. A function $f$ is in $\mathcal{T}_{L}^{k}$ if and only if, for any pair of integers $(n, m)$ with $w(n, m) \leq k$ and $j \in I^{n}$, the Bruhat distributions $X^{j} L^{m} f$ can be represented by continuous functions and $x \mapsto\left|D_{x}^{n} L^{m} f\right|_{L}$ is continuous on $G$.

\section{Heat kernel Derivative estimates}

4.1. Gaussian estimates for derivatives. Fix a central symmetric Gaussian semigroup $\left(\mu_{t}\right)_{t>0}$ on $G$ with infinitesimal generator $-L$.

Definition 4.1. Let $\rho: G \times G \rightarrow[0,+\infty)$ be a bi-invariant continuous distance function on $G$ and set $\rho(x)=\rho(e, x)$. We say that $\rho$ is adapted to $L$ (equivalently, to $\left.\left(\mu_{t}\right)_{t>0}\right)$ if it has the following property: for any non-negative function $\phi \in \mathcal{B}(G)$ such that $\int \phi d \nu=1, \rho * \phi$ satisfies

$$
\Gamma(\rho * \phi, \rho * \phi)=\sum_{i}\left|X_{i}(\rho * \phi)\right|^{2} \leq 1 .
$$

Examples will be given in Section 4.2

Theorem 4.2. Let $\left(\mu_{t}\right)_{t>0}$ be a central symmetric Gaussian semigroup on $G$ with infinitesimal generator $-L$. Let $X=(X)_{I}$ be a projective family such that $-L=$ $\sum_{i \in I} X_{i}^{2}$.

1. Assume that $\left(\mu_{t}\right)_{t>0}$ satisfies property $(\mathrm{CK})$. Then, for all $t>0$, the continuous density $x \mapsto \mu_{t}(x)$ of the measure $\mu_{t}$ belongs to $\mathcal{T}_{L}$.

2. Let $\rho$ be an adapted distance. Assume that $\left(\mu_{t}\right)_{t>0}$ satisfies $(\mathrm{CK})$. Assume further that there is a positive decreasing continuous function $M(t)$ and a constant $B_{0}>0$ such that

$$
\forall t \in(0,1), \quad \forall x \in G, \quad \mu_{t}(x) \leq \exp \left(M(t)-\frac{\rho(x)^{2}}{B_{0} t}\right) .
$$

Then, for any $(k, n) \in \mathbb{N}^{2}$, there exist positive constants $A, B, C$, a such that

$$
\forall t \in(0,1), \quad \forall x \in G, \quad\left|D_{x}^{k} L^{n} \mu_{t}\right|_{L} \leq C t^{-n-k / 2} \exp \left(A M(a t)-\frac{\rho(x)^{2}}{B t}\right) .
$$

Proof of " $\mu_{t} \in \mathcal{T}_{L}$ ". We start with the following simple lemma.

Lemma 4.3. For any $f \in \mathcal{B}(G)$,

$$
\int f\left(L^{k} f\right) d \nu=\int\left|D^{k} f\right|_{L}^{2} d \nu
$$

Proof. To see this observe that

$$
\begin{aligned}
\int f\left(L^{k} f\right) d \nu & =-\int f\left[\sum_{i \in I} X_{i}^{2} L^{k-1} f\right] d \nu \\
& =\sum_{i \in I} \int\left[X_{i} f\right]\left[X_{i} L^{k-1} f\right] d \nu=\sum_{i \in I} \int\left[X_{i} f\right]\left[L^{k-1} X_{i} f\right] d \nu
\end{aligned}
$$

The lemma follows by induction.

Remark 4.4. The lemma above depends heavily on the fact that $L$ is central, i.e., commutes with any left-invariant vector field. In general, the correct statement is in terms of iterated gradients. Namely, $\int f\left(L^{k} f\right) d \nu=\int \Gamma_{k}(f, f) d \nu$. See [25]. 
Lemma 4.5. Let $\left(\mu_{t}\right)_{t>0}$ be a central symmetric Gaussian semigroup on $G$ satisfying $(\mathrm{CK})$. Then $\mu_{t} \in \mathcal{T}_{L}$ and, for any pair of integers $(n, m)$, we have

$$
\left|D_{x}^{n} L^{m} \mu_{t}\right|_{L} \leq n^{n / 2} m^{m}\left(\frac{4}{t}\right)^{w(n, m) / 2} \mu_{t / 2}(e) .
$$

Proof. As $\mathcal{B}(G)$ is dense in the $L^{2}(G, d \nu)$-domain $\mathcal{D}_{2}^{2 k}$ of $L^{k}$, the identity of Lemma 4.3 extends to any function in $\mathcal{D}_{2}^{2 k}$. In particular,

$$
\left\|\left|D^{n} L^{m} \mu_{t}\right|_{L}\right\|_{2}^{2}=\int\left(L^{m} \mu_{t}\right)\left(L^{n+m} \mu_{t}\right) d \nu=\left\|L^{m+n / 2} \mu_{t}\right\|_{2}^{2} .
$$

Let $H_{t}=e^{-t L}$ be the semigroup of operators defined at (1.1). Then, for any $f \in L^{2}(G, \nu)$,

$$
2\left\|L^{1 / 2} H_{t} f\right\|_{2}^{2}=2\left\langle L H_{t} f, H_{t} f\right\rangle=-\partial_{t}\left\|H_{t} f\right\|_{2}^{2}
$$

is a non-negative decreasing function. As

$$
2 \int_{0}^{t}\left\langle L H_{s} f, H_{s} f\right\rangle d s=\|f\|_{2}^{2}-\left\|H_{t} f\right\|_{2}^{2}
$$

it follows that

$$
2 t\left\|L^{1 / 2} H_{t} f\right\|_{2}^{2} \leq\|f\|_{2}^{2}
$$

In other words,

$$
\left\|L^{1 / 2} H_{t}\right\|_{2 \rightarrow 2} \leq\left(\frac{1}{2 t}\right)^{1 / 2} .
$$

By the semigroup property, this implies

$$
\left\|L^{k / 2} H_{t}\right\|_{2 \rightarrow 2} \leq\left(\frac{k}{2 t}\right)^{k / 2}
$$

and

$$
\begin{aligned}
\left\|L^{k / 2} \mu_{t}\right\|_{2}^{2} & =\left\|L^{k / 2} H_{t}\right\|_{2 \rightarrow \infty}^{2} \leq\left\|L^{k / 2} H_{t / 2}\right\|_{2 \rightarrow 2}^{2}\left\|H_{t / 2}\right\|_{2 \rightarrow \infty}^{2} \\
& \leq\left(\frac{k}{t}\right)^{k} \mu_{t}(e) .
\end{aligned}
$$

Finally, for any integer $p, q$, we obtain

$$
\left\|\left|D^{p} L^{q / 2} \mu_{t}\right|_{L}\right\|_{2}^{2}=\left\|L^{(p+q) / 2} \mu_{t}\right\|_{2}^{2} \leq\left(\frac{p+q}{t}\right)^{p+q} \mu_{t}(e) .
$$

To prove Lemma 4.5, use the semigroup property once more and write

$$
\begin{aligned}
\left|D_{x}^{n} L^{m} \mu_{t}\right|_{L}^{2} & =\sum_{\ell \in I^{n}}\left|X^{\ell} \mu_{t / 2} * L^{m} \mu_{t / 2}(x)\right|^{2} \\
& =\sum_{\ell \in I^{n}}\left|\int X^{\ell} \mu_{t / 2}\left(y^{-1} x\right) L^{m} \mu_{t / 2}(y) d \nu(y)\right|^{2} \\
& \leq \sum_{\ell \in I^{n}}\left\|X^{\ell} \mu_{t / 2}\right\|_{2}^{2}\left\|L^{m} \mu_{t / 2}\right\|_{2}^{2} \\
& =\left\|\left|D^{n} \mu_{t / 2}\right|_{L}\right\|_{2}^{2}\left\|L^{m} \mu_{t / 2}\right\|_{2}^{2} \leq n^{n} m^{2 m}\left(\frac{4}{t}\right)^{n+2 m} \mu_{t / 2}(e)^{2} .
\end{aligned}
$$


To see that $x \mapsto X^{\ell} L^{m} f(x), \ell \in I^{n}$, and $x \mapsto\left|D_{x}^{n} L^{m} f\right|_{L}$ are continuous, observe that both $\left|X^{\ell} L^{m} f(x)-X^{\ell} L^{m} f(y)\right|^{2}$ and $\left.|| D_{x}^{n} L^{m} \mu_{t}\right|_{L}-\left.\left|D_{y}^{n} L^{m} \mu_{t}\right|_{L}\right|^{2}$ are bounded by

$$
\begin{aligned}
\sum_{\ell \in I^{n}} \mid X^{\ell} L^{m} \mu_{t / 2} * & \mu_{t / 2}(x)-\left.X^{\ell} \mu_{t / 2} * \mu_{t / 2}(y)\right|^{2} \\
& \leq \sum_{\ell \in I^{n}}\left(\int\left|X^{\ell} L^{m} \mu_{t / 2}(z)\right| \mid\left(\mu_{t / 2}\left(x z^{-1}\right)-\mu_{t / 2}\left(y z^{-1}\right) \mid\right)^{2} d \nu(z)\right. \\
& \leq\left\|\left|D^{n} L^{m} \mu_{t / 2}\right|_{L}\right\|_{2}^{2} \sup _{z \in G}\left|\mu_{t / 2}\left(x z^{-1}\right)-\mu_{t / 2}\left(y z^{-1}\right)\right|^{2}
\end{aligned}
$$

This, together with finiteness of $\left\|\left|D^{n} L^{m} \mu_{t / 2}\right|_{L}\right\|_{2}$ and uniform continuity of the density $\mu_{t}$, shows that $x \mapsto X^{\ell} L^{m} \mu_{t}(x), \ell \in I^{n}$ and $x \rightarrow\left|D_{x}^{n} L^{m} \mu_{t}\right|_{L}$ are continuous.

Clearly, Lemma 4.5 shows that $\mu_{t} \in \mathcal{T}_{L}$.

Proof of the Gaussian upper-bounds. We start with the following lemma.

Lemma 4.6. Under the hypothesis of Theorem 4.2, we have

$$
\forall t \in(0,1), \quad \forall x \in G, \quad\left|L^{k} \mu_{t}(x)\right| \leq(18)^{k} k ! t^{-k} \exp \left(M(t / 2)-\frac{2 \rho(x)^{2}}{3 B_{0} t}\right) .
$$

Proof. After observing that $(-L)^{k} \mu_{t}=\partial_{t}^{k} \mu_{t}$, this follows from the hypotheses and [17, Theorem 4] by taking $y=e, \delta=1 / 2, \epsilon=1 / 9, a=b=\exp (M(t))$, $c=\exp \left(-\rho(x)^{2} /\left[B_{0} t\right]\right)$ in that theorem.

Thus we are left with proving the corresponding bounds for

$$
\left|D_{x}^{k} L^{n} \mu_{t}\right|_{L}, \quad k=1,2, \ldots, \quad n=0,1, \ldots
$$

We claim it suffices to prove that, for any $k=0,1, \ldots, n=0,1, \ldots$, there exist positive constants $A, a, B, C$ (depending on $(n, k))$ such that

$$
\forall \alpha>0, \quad \forall t \in(0,1), \quad\left\|e^{\alpha \rho}\left|D^{k} L^{n} \mu_{t}\right|_{L}\right\|_{\infty} \leq C t^{-n-k / 2} \exp \left(A M(a t)+B \alpha^{2} t\right) .
$$

Indeed, given (4.1), write

$$
\left|D_{x}^{k} L^{n} \mu_{t}\right|_{L} \leq C t^{-n-k / 2} \exp \left(A M(a t)+B \alpha^{2} t-\alpha \rho(x)\right)
$$

and choose $\alpha=\rho(x) /[2 B t]$. This yields

$$
\left|D_{x}^{k} L^{n} \mu_{t}\right|_{L} \leq C t^{-n-k / 2} \exp \left(A M(a t)-\frac{\rho(x)^{2}}{4 B t}\right)
$$

as desired.

Next, we claim that it suffices to prove that, for any $k=0,1, \ldots, n=0,1, \ldots$, there exist positive constants $A, a, B, C$ such that

$$
\forall \alpha>0, \forall t \in(0,1),\left\|e^{\alpha \rho}\left|D^{k} L^{n} \mu_{t}\right|_{L}\right\|_{2} \leq C t^{-n-k / 2} \exp \left(A M(a t)+B \alpha^{2} t\right) .
$$


Indeed, assuming that (4.2) holds and using the triangle inequality $\rho(x) \leq \rho\left(y^{-1} x\right)+$ $\rho(y)$, we have

$$
\begin{aligned}
& e^{2 \alpha \rho(x)}\left|D_{x}^{k} L^{n} \mu_{t}\right|_{L}^{2} \\
& \quad \leq \sum_{\ell \in I^{k}}\left|\int\left[e^{\alpha \rho\left(y^{-1} x\right)} X^{\ell} L^{n} \mu_{t / 2}\left(y^{-1} x\right)\right]\left[e^{\alpha \rho(y)} \mu_{t / 2}(y)\right] d \nu(y)\right|^{2} \\
& \quad \leq\left\|e^{\alpha \rho}\left|D^{k} L^{n} \mu_{t / 2}\right|_{L}\right\|_{2}^{2}\left\|e^{\alpha \rho} \mu_{t / 2}\right\|_{2}^{2} .
\end{aligned}
$$

By the postulated Gaussian upper-bound on $\mu_{t}$ and the elementary inequality

$$
\forall \alpha, b, t, \rho>0, \quad \alpha \rho-\frac{\rho^{2}}{b t} \leq \frac{\alpha^{2} b t}{4}
$$

we have

$$
\left\|e^{\alpha \rho} \mu_{t / 2}\right\|_{2}^{2} \leq \exp \left(2 M(t / 2)+\frac{\alpha^{2} B_{0} t}{4}\right)
$$

Thus (4.2) implies (4.1) as claimed.

In order to prove (4.2) we will proceed by induction on $k$. By Lemma 4.6 and (4.3), the upper-bound (4.2) is satisfied for $k=0$. Assume it is satisfied for some integer $k$. Fix a non-negative function $\phi \in \mathcal{B}(G)$ and let $\varrho=\phi * \rho$. Then write

$$
\begin{aligned}
& \int e^{2 \alpha \varrho}\left|D^{k+1} L^{n} \mu_{t}\right|_{L}^{2} d \nu= \sum_{\ell \in I^{k+1}} \int e^{2 \alpha \varrho}\left|X^{\ell} L^{n} \mu_{t}\right|^{2} d \nu \\
&= \sum_{i \in I} \sum_{\ell \in I^{k}} \int e^{2 \alpha \varrho}\left|X_{i} X^{\ell} L^{n} \mu_{t}\right|^{2} d \nu \\
&=-\sum_{i \in I} \sum_{\ell \in I^{k}} \int\left(\left[X_{i} e^{2 \alpha \varrho}\right]\left[X^{\ell} L^{n} \mu_{t}\right]\left[X_{i} X^{\ell} L^{n} \mu_{t}\right]\right. \\
&\left.+e^{2 \alpha \varrho}\left[X^{\ell} L^{n} \mu_{t}\right]\left[X_{i}^{2} X^{\ell} L^{n} \mu_{t}\right]\right) d \nu \\
&=-2 \alpha \sum_{\ell \in I^{k}} \int e^{2 \alpha \varrho}\left[\sum_{i}\left(X_{i} \varrho\right)\left(X_{i} X^{\ell} L^{n} \mu_{t}\right)\right]\left[X^{\ell} L^{n} \mu_{t}\right] d \nu \\
&+\sum_{\ell \in I^{k}} \int e^{2 \alpha \varrho}\left[X^{\ell} L^{n} \mu_{t}\right]\left[L X^{\ell} L^{n} \mu_{t}\right] d \nu \\
& \leq 2 \alpha \sum_{\ell \in I^{k}} \int e^{2 \alpha \varrho}\left(\sum_{i}\left|X_{i} \varrho \| X_{i} X^{\ell} L^{n} \mu_{t}\right|\right)\left|X^{\ell} L^{n} \mu_{t}\right| d \nu \\
&+\sum_{\ell \in I^{k}} \int e^{2 \alpha \varrho}\left|X^{\ell} L^{n} \mu_{t}\right|\left|X^{\ell} L^{n+1} \mu_{t}\right| d \nu \\
&= 2 \alpha E_{1}+E_{2} .
\end{aligned}
$$

Note that to obtain the formula which gives $E_{2}$ we have used the fact that $L$ commutes with any left-invariant vector field. Next, recall that our hypothesis on 
$\rho$ implies that $\sum_{i}\left|X_{i} \varrho\right|^{2} \leq 1$ and write

$$
\begin{aligned}
E_{1} & =\sum_{\ell \in I^{k}} \int e^{2 \alpha \varrho}\left(\sum_{i}\left|X_{i} \varrho\right|\left|X_{i} X^{\ell} L^{n} \mu_{t}\right|\right)\left|X^{\ell} L^{n} \mu_{t}\right| d \nu \\
& \leq \int e^{2 \alpha \varrho} \sum_{\ell \in I^{k}}\left(\sum_{i}\left|X_{i} X^{\ell} L^{n} \mu_{t}\right|^{2}\right)^{1 / 2}\left|X^{\ell} L^{n} \mu_{t}\right| d \nu \\
& \leq \int e^{2 \alpha \varrho}\left(\sum_{\ell \in I^{k}} \sum_{i}\left|X_{i} X^{\ell} L^{n} \mu_{t}\right|^{2}\right)^{1 / 2}\left(\sum_{\ell \in I^{k}}\left|X^{\ell} L^{n} \mu_{t}\right|^{2}\right)^{1 / 2} d \nu \\
& \leq\left(\int \sum_{\ell \in I^{k+1}}\left|X^{\ell} L^{n} \mu_{t}\right|^{2} d \nu\right)^{1 / 2}\left(\int e^{4 \alpha \varrho} \sum_{\ell \in I^{k}}\left|X^{\ell} L^{n} \mu_{t}\right|^{2} d \nu\right)^{1 / 2} \\
& \leq\left\|\left|D^{k+1} L^{n} \mu_{t}\right|_{L}\right\|_{2}\left\|e^{2 \alpha \varrho}\left|D^{k} L^{n} \mu_{t}\right|_{L}\right\|_{2} .
\end{aligned}
$$

To bound $E_{2}$, write

$$
E_{2} \leq\left\|e^{\alpha \varrho}\left|D^{k} L^{n} \mu_{t}\right|_{L}\right\|_{2}\left\|e^{\alpha \varrho}\left|D^{k} L^{n+1} \mu_{t}\right|_{L}\right\|_{2} .
$$

By (4.4), (4.5), (4.6), the induction hypothesis and Lemma 4.5, we obtain

$$
\begin{aligned}
\left\|e^{\alpha \varrho}\left|D^{k+1} L^{n} \mu_{t}\right|_{L}\right\|_{2}^{2} \leq & 2 \alpha\left\|\left|D^{k+1} L^{n} \mu_{t}\right|_{L}\right\|_{2}\left\|e^{2 \alpha \varrho}\left|D^{k} L^{n} \mu_{t}\right|_{L}\right\|_{2} \\
& +\left\|e^{\alpha \varrho}\left|D^{k} L^{n} \mu_{t}\right|_{L}\right\|_{2}\left\|e^{\alpha \varrho}\left|D^{k} L^{n+1} \mu_{t}\right|_{L}\right\|_{2} \\
\leq & C\left(1+2 \alpha t^{1 / 2}\right) t^{-2 n-k-1} \exp \left(A M(a t)+B \alpha^{2} t\right) \\
\leq & C^{\prime} t^{-2 n-k-1} \exp \left(A M(a t)+B^{\prime} \alpha^{2} t\right) .
\end{aligned}
$$

This finishes the inductive proof of (4.2) and the proof of Theorem 4.2

4.2. Examples of Gaussian estimates. Let us fix a symmetric central Gaussian semigroup $\left(\mu_{t}\right)_{t>0}$. Given a $\lambda>0$, we say that $\left(\mu_{t}\right)_{t>0}$ satisfies $(\mathrm{CK} \lambda)$ if property (CK) holds and the continuous density $\mu_{t}(x)$ satisfies

$$
\kappa=\sup _{0<t<1}\left\{t^{\lambda} \log \mu_{t}(e)\right\}<+\infty .
$$

In order to apply Theorem 4.2 we need to have some basic Gaussian estimates for the density $\mu_{t}(x)$ of our Gaussian semigroup in terms of some bi-invariant distance adapted to $L$. The next definition provides adapted distance candidates.

Definition 4.7. Given a symmetric Gaussian semigroup $\left(\mu_{t}\right)_{t>0}$ on $G$ with infinitesimal generator $-L$, we set

$$
d(x, y)=d_{L}(x, y)=\sup \{|f(x)-f(y)|: f \in \mathcal{B}(G), \quad \Gamma(f, f) \leq 1\}
$$

and

$$
\delta(x, y)=\delta_{L}(x, y)=\sup \{|f(x)-f(y)|: f \in \mathcal{B}(G), \quad \Gamma(f, f) \leq 1, \quad|L f| \leq 1\} .
$$

These quasi-distances are called, respectively the intrinsic distance and the relaxed distance associated with $L$.

The distances $d$ and $\delta$ are not necessarily adapted because it may happen that they are not continuous. However, if $d$ (resp. $\delta$ ) is continuous, then it is not hard to show that it is adapted. Indeed, if $d$ (resp. $\delta$ ) is continuous, then it satisfies $\Gamma(d, d) \leq 1(\operatorname{resp} . \Gamma(\delta, \delta) \leq 1)$ almost everywhere. 
Gaussian estimates involving either the intrinsic distance $d$ or the relaxed distance $\delta$ introduced in Definition 4.7 have been obtained in [6] under various hypotheses. We now recall these results which are crucial in the sequel.

The following result is taken from [6, 7].

Theorem 4.8. Let $\left(\mu_{t}\right)_{t>0}$ be a symmetric Gaussian semigroup satisfying property (CK) and let $x \mapsto \mu_{t}(x)$ be its continuous density.

(1) Assume that $\left(\mu_{t}\right)_{t>0}$ satisfies $(\mathrm{CK} *)$. Then the relaxed distance $\delta$ is a continuous distance function which defines the topology of $G$ and

$$
\forall t \in(0,1), \forall x \in G, \quad \mu_{t}(x) \leq \exp \left(M(t)-\frac{\delta(x)^{2}}{C t}\right)
$$

where $M$ satisfies $\lim _{t \rightarrow 0} t M(t)=0$.

(2) Fix $\lambda \in(0,1)$. Assume that $\left(\mu_{t}\right)_{t>0}$ satisfies $(\mathrm{CK} \lambda)$. Then the intrinsic distance $d$ is a continuous distance function which defines the topology of $G$ and

$$
\forall t \in(0,1), \forall x \in G, \quad \mu_{t}(x) \leq \exp \left(\frac{A}{t^{\lambda}}-\frac{d(x)^{2}}{C t}\right) .
$$

Let us comment that, in Theorem 4.8(1), the intrinsic distance $d$ might well be equal to $+\infty$ almost everywhere in which case no Gaussian estimate involving the intrinsic distance can possibly hold. Thus the relaxed distance plays a crucial role in this case.

Applying Theorem 4.2 and the above result we obtain the following corollary.

Corollary 4.9. Let $d, \delta$ denote the intrinsic and relaxed distances, respectively.

(1) Assume that $\left(\mu_{t}\right)_{t>0}$ satisfies (CK*). Then, for each fixed $k$ and $n$ there exists $C=C(k, n)$ such that

$$
\forall t \in(0,1), \forall x \in G, \quad\left|D_{x}^{k} L^{n} \mu_{t}\right|_{L} \leq \exp \left(M(t)-\frac{\delta(x)^{2}}{C t}\right)
$$

where $M$ satisfies $\lim _{t \rightarrow 0} t M(t)=0$.

(2) Fix $\lambda \in(0,1)$. Assume that $\left(\mu_{t}\right)_{t>0}$ satisfies $(\mathrm{CK} \lambda)$ and let $\kappa$ be as in (4.7). Then, for each fixed $k$ and $n$ there exists $A=A(\kappa, \lambda, k, n)$ and $C=C(k, n)$ such that,

$$
\forall t \in(0,1), \forall x \in G, \quad\left|D_{x}^{k} L^{n} \mu_{t}\right|_{L} \leq \exp \left(\frac{A}{t^{\lambda}}-\frac{d(x)^{2}}{C t}\right) .
$$

In terms of potential theory, the importance of condition $(\mathrm{CK} *)$ and of the Gaussian bound stated in Theorem 4.8(1) is that it implies that

$$
\lim _{t \rightarrow 0} \sup _{x \in K} \mu_{t}(x)=0
$$

for any compact $K$ which does not contain $e$. See [4, [5, 6] where we call this property (CK\#). The next corollary gives a bound on the Green function $g=\int_{0}^{\infty} e^{-t} \mu_{t} d t$. As defined, $g$ is a measure. In [8], it is proved that $g$ is absolutely continuous w.r.t. Haar measure and admits a continuous density on $G \backslash\{e\}$ if and only if property $(\mathrm{CK} *)$ holds true. In this case, we denote by $x \mapsto g(x)$ the continuous density of $g$ on $G \backslash\{e\}$. The following result easily follows from the bounds of Corollary 4.9 . The proof is omitted. 
Corollary 4.10. Let $\left(\mu_{t}\right)_{t>0}$ be a symmetric central Gaussian semigroup.

(1) Assume that $\left(\mu_{t}\right)_{t>0}$ satisfies $(\mathrm{CK} *)$. Then, for any function $\phi \in \mathcal{B}(G)$ with support in $G \backslash\{e\}, \phi g$ belongs to $\mathcal{T}_{L}$. In particular, for any integers $n, m$ and $j \in I^{m}$, the Bruhat distribution $X^{j} L^{n} g$ can be represented in $G \backslash\{e\}$ by a continuous function.

(2) Fix $\lambda \in(0,1)$. Assume that $\left(\mu_{t}\right)_{t>0}$ satisfies $(\mathrm{CK} \lambda)$. Then, for any fixed integers $n, m$, there exists a constant $C$ such that

$$
\forall x \in G \backslash\{e\}, \quad \log \left(\left|D_{x}^{m} L^{n} g\right|_{L}\right) \leq C d(x)^{-\frac{2 \lambda}{1-\lambda}} .
$$

In [11], in order to study hypoellipticity questions, we will use the following result which is in the same spirit as (4.8). For each $\Omega \subset G$ and $f \in \mathcal{T}_{L}$, set

$$
M_{L}^{k}(\Omega, f)=\sup _{x \in \Omega} \sup _{\substack{(n, m) \in \mathbb{N} \\ w(n, m) \leq k}}\left\{\left|D_{x}^{n} L^{m} f\right|_{L}\right\} .
$$

Corollary 4.11. Assume that $\left(\mu_{t}\right)_{t>0}$ is a symmetric central Gausssian semigroup satisfying condition $(\mathrm{CK} *)$. Then, for any compact set $K$ with $e \notin K$, any integer $k$, any $\sigma>0$, there exists a constant $C$ (which depends on $\left(\mu_{t}\right)_{t>0}, K, k$ and $\sigma$ ) such that

$$
\sup _{t \in(0,1)}\left\{t^{-\sigma} M_{L}^{k}\left(K, \mu_{t}\right)\right\} \leq C .
$$

Proof. Under condition $(\mathrm{CK} *)$ the relaxed distance is continuous and defines the topology of $G$. As $K$ is compact and does not contain $e$ it follows that $\inf _{K} \delta(x)>0$. The desired result thus follows from Corollary 4.9

Example 4.12. Let $G=\mathbf{T}=\mathbf{R} / 2 \pi \mathbf{Z}$ where $\mathbf{R}=\mathbb{R}^{\infty}$ and $\mathbf{Z}=\mathbb{Z}^{\infty}$, as in the example of Section 2.3 Write $\mathbf{T}=(\mathbb{R} / 2 \pi \mathbb{Z})^{\infty}$ as an infinite product of circles and consider the projective basis $Y=\left(Y_{i}\right)_{1}^{\infty}$ where $Y_{i}=\partial_{i}$ is identified with partial differentiation in the $i$-th coordinate. For any sequence $a=\left(a_{i}\right), a_{i}>0$, let $\left(\mu_{t}^{a}\right)_{t>0}$ the symmetric Gaussian semigroup with generator $-L=\sum a_{i} \partial_{i}^{2}$. Set $N_{a}(s)=$ $\#\left\{i: a_{i} \leq s\right\}$. Then, $\left(\mu_{t}^{a}\right)_{t>0}$ satisfies (CK*) if and only if $N_{a}(s)=o(s)$ as $s$ tends to infinity. For any fixed $\lambda>0,\left(\mu_{t}^{a}\right)_{t>0}$ satisfies $(\mathrm{CK} \lambda)$ if and only if $N_{a}(s)=O\left(s^{\lambda}\right)$ as $s$ tends to infinity. See [2, 3, 6]. Thus, assuming that $N_{a}(s)=O\left(s^{\lambda}\right)$ at infinity, for some $\lambda \in(0,1)$, Corollary 4.9 gives the following bound on the first order spatial derivatives:

$$
\forall t \in(0,1), \quad \forall x \in \mathbf{T}, \quad \sum_{i} a_{i}\left|\partial_{i} \mu_{t}^{a}(x)\right|^{2} \leq \exp \left(A t^{-\lambda}-\frac{d(x)^{2}}{C t}\right) .
$$

Example 4.13. Keeping the notation of Example 4.12, consider an increasing sequence $b=\left(b_{i}\right)$ of positive numbers. Set

$$
A=\left(\begin{array}{cccccccc}
b_{1} & b_{1} & b_{1} & b_{1} & b_{1} & b_{1} & \cdot & \cdot \\
b_{1} & b_{2} & b_{2} & b_{2} & b_{2} & b_{2} & \cdot & \cdot \\
b_{1} & b_{2} & b_{3} & b_{3} & b_{3} & b_{3} & \cdot & \cdot \\
b_{1} & b_{2} & b_{3} & b_{4} & b_{4} & b_{4} & \cdot & \cdot \\
b_{1} & b_{2} & b_{3} & b_{4} & b_{5} & b_{5} & \cdot & \cdot \\
b_{1} & b_{2} & b_{3} & b_{4} & b_{5} & b_{6} & \cdot & \cdot \\
\cdot & \cdot & \cdot & \cdot & \cdot & \cdot & \cdot & \cdot \\
\cdot & \cdot & \cdot & \cdot & \cdot & \cdot & \cdot & \cdot
\end{array}\right)
$$


This generalizes the matrix $A_{1}$ of the example of Section 2.3 which corresponds to $b_{i}=i$ for all $i$. Thus, $A=\left(a_{i, j}\right)$ with $a_{i, j}=\min \left\{b_{i}, b_{j}\right\}$. Set $X_{i}=\sum_{j \geq i} Y_{i}$. Then the family $\left(X_{i}\right)_{1}^{\infty}$ is a projective basis of the Lie algebra $\mathbf{R}$ of $\mathbf{T}$. Moreover, as observed in Section 2.3, $\mathbf{Z}_{X}=\mathbf{Z}$ so that $\mathbf{T}$ is in fact the direct product of the circles given by the one parameter subgroup generated by the $X_{i}$ 's. Let $\left(\mu_{t}\right)_{t>0}$ be the symmetric Gaussian semigroup with infinitesimal generator $-L=\sum_{i, j} a_{i, j} Y_{i} Y_{j}$. In the basis $X$, we have $-L=\sum_{i} u_{i} X_{i}^{2}$ where $u_{1}=b_{1}$ and $u_{i}=b_{i}-b_{i-1}$ for $i \geq 2$. Thus $\left(\mu_{t}\right)_{t>0}$ satisfies $(\mathrm{CK} *)$ if and only if $\#\left\{u_{i} \leq s\right\}=o(s)$ at infinity. For instance, this is the case if $b_{i}=i^{2} \log (1+i)$. $\left(\mu_{t}\right)_{t>0}$ satisfies $(\mathrm{CK} \lambda)$ if and only if $\#\left\{u_{i} \leq s\right\}=O\left(s^{\lambda}\right)$ at infinity, e.g., if $b_{i}=i^{1+1 / \lambda}$. Corollary 4.9 gives Gaussian upper-bounds for derivatives in the directions of the basis $X$ but, since $\partial_{i}=Y_{i}=X_{i}-X_{i+1} \in \mathcal{H}_{L}$, one easily deduces Gaussian bounds in the directions of the basis $Y$. For instance, assuming that $b_{i}=i^{1+1 / \lambda}$ for some fixed $\lambda \in(0,1)$, we obtain

$$
\forall i, \forall t \in(0,1), \forall x \in \mathbf{T}, \quad i^{1 / \lambda}\left|\partial_{i} \mu_{t}(x)\right| \leq \exp \left(A t^{-\lambda}-\frac{d(x)^{2}}{C t}\right) .
$$

Example 4.14. A compact connected group is semisimple if it is equal to its commutator subgroup. See [21]. For any semisimple group $G$, there exists a family $\left(\Sigma_{k}\right)$ of compact connected simple Lie groups, and a closed central subgroup $H$ of $\Sigma=\prod \Sigma_{k}$ such that $G=\Sigma / H$. Since we assume that $G$ is metrizable, the family $\left(\Sigma_{k}\right)$ is countable. The center of $\Sigma$, being a product of finite groups, is totally disconnected. Thus, so is $H$. It follows that $\Sigma$ and $G$ have the same Lie algebra (see [8]). The infinitesimal generator $-L$ of any given symmetric central Gaussian semigroup $\left(\mu_{t}\right)_{t>0}$ on $G$ has the form $-L=\sum a_{k} \Delta_{k}$ where $a_{k} \geq 0$ and $\Delta_{k}$ is the Laplace-Beltrami operator of the canonical Killing metric on $\Sigma_{k}$ (i.e., the Casimir operator). Let also $\left|\nabla_{k} f\right|_{k}$ denote the length of the gradient in the Killing metric on $\Sigma_{k}$. In what follows we assume that $L$ is not degenerate, i.e., $a_{k}>0$ for all $k$. Set

$$
N(s)=\sum_{a_{k} \leq s} n_{k}
$$

where $n_{k}$ is the topological dimension of $\Sigma_{k}$. Then $\left(\mu_{t}\right)_{t>0}$ satisfies $(\mathrm{CK} *)$ if and only if $N(s)=o(s)$ as $s$ tends to infinity. It satisfies $(\mathrm{CK} \lambda)$ if and only if $N(s)=$ $O\left(s^{\lambda}\right)$. See 4 [ 8]. Assuming that $N(s)=O\left(s^{\lambda}\right)$ for some $\lambda \in(0,1)$, we obtain that

$$
\sum_{k} a_{k}\left|\nabla_{k} \mu_{t}(x)\right|_{k}^{2} \leq \exp \left(A t^{-\lambda}-\frac{d(x)^{2}}{C t}\right)
$$

and similar estimates for higher derivatives.

\section{REFERENCES}

1. Bakry D. Transformation de Riesz pour les semigroupes symétriques. Séminaire de Probabilités XIX, Lect. Notes in Math. 1123, Springer, 1985, 130-174. MR 89h:42023

2. Bendikov A. Potential Theory on Infinite Dimensional Abelian Groups. Walter De Gruyter \& Co., Berlin-New York, 1995. MR 98i:31007

3. Bendikov A. Symmetric stable semigroups on the infinite dimensional torus. Expo. Math., 13, 1995, 39-79. MR 96h:60123

4. Bendikov A. and Saloff-Coste L. Elliptic diffusions on infinite products. J. reine angew. Math. 493, 1997, 171-220. MR 99k:58173

5. Bendikov A. and Saloff-Coste L. Potential theory on infinite products and groups. Potential Analysis, 11, 325-358, 1999. MR 2001a:31012 
6. Bendikov A. and Saloff-Coste L. On and off-diagonal heat kernel behaviors on certain infinite dimensional local Dirichlet spaces. American J. Math. 122, 2000, 1205-1263. CMP 2001:05

7. Bendikov A. and Saloff-Coste L. Gaussian semigroups of measures on locally compact locally connected groups, Preprint 1999.

8. Bendikov A. and Saloff-Coste L. Central Gaussian semigroups of measures with continuous density, J. Funct. Anal. to appear.

9. Bendikov A. and Saloff-Coste L. On the absolute continuity of Gaussian measures on locally compact groups, J. Theoret. Prob. to appear.

10. Bendikov A. and Saloff-Coste L. On the hypoellipticity of sub-Laplacians on infinite dimensional compact groups. Forum Math. to appear.

11. Bendikov A. and Saloff-Coste L. Hypoellipticity for bi-invariant Laplacians on infinite dimensional compact groups. Preprint 2001.

12. Berg Ch. Potential theory on the infinite dimensional torus. Invent. Math. 32, 1976, 49-100. MR 53:5915

13. Born E. Projective Lie Algebra Bases of a Locally Compact Group and Uniform Differentiability. Math. Zeit. 200, 1989, 279-292. MR 90f:22008

14. Born E. An Explicit Lévy-Hinčin Formula for Convolution Semigroups on Locally Compact Groups. J. Theor. Prob. 2, 1989, 325-342. MR 91a:60023

15. Bruhat F. Distributions sur un groupe localement compact et application à l'étude des représentations des groupes p-adiques. Bull. Soc. Math. France, 89, 1961, 43-75. MR 25:4354

16. Davies E.B. Heat kernels and spectral theory. 1989, Cambridge University Press. MR 90e:35123

17. Davies E.B. Non-Gaussian aspects of heat kernel behaviour. J. London Mat. Soc. 55, 1997, 105-125. MR 97i:58169

18. Fukushima M., Oshima Y. and Takeda, M. Dirichlet forms and symmetric Markov processes, W. De Gruyter, 1994. MR 96f:60126

19. Glushkov V.N. The structure of locally compact groups and Hilbert's Fifth Problem. AMS Translations 15, 1960, 55-94. MR 22:5690

20. Heyer H. Probability Measures on Locally Compact Groups. Ergeb. der Math. und ihrer Grenzgeb. 94, Springer, Berlin-Heildelberg-New York, 1977. MR 58:18648

21. Hofmann K. and Morris S. The structure of compact groups. W. de Gruyter, 1998. MR 99k:22001

22. Hörmander L. Hypoelliptic second order differential equations. Acta Math. 119, 1967, 147-171. MR 36:5526

23. Hunt G. A. Semi-groups of measures on Lie groups. Trans. A.M.S., 81, 1956, 264-293. MR 18:54a

24. Kusuoka S. and Stroock D. Applications of the Malliavin calculus, Part II. J. Fac. Sci., The University of Tokyo, Sec IA, 32, 1985, 1-76. MR 86 k:60100b

25. Ledoux M. L'algèbre des gradients itérés d'un générateur Markovien - Développements de moyennes et entropies. Ann. scient. Éc. Norm. Sup. 4ème série, 28, 1995, 435-460. MR 96f: 60130

26. Saloff-Coste L. Analyse sur les groupes de Lie nilpotents. C. R. Acad. Sci. Paris, 302, Série I, 1986, 499-502. MR 88j:22008

27. Siebert E. Absolute continuity, singularity and supports of Gaussian semigroups on a Lie group. Monatshefte für Math. 93, 1982, 239-253. MR 83m:60018

28. Sturm K-T. On the geometry defined by Dirichlet forms. In Seminar on Stochastic Processes, Random Fields and Applications, Ascona, E. Bolthausen et al., Eds. Birkhäuser, Progress in Probability, Vol 36, 1995, 231-242. MR 96j:31009

29. Varopoulos N., Saloff-Coste L. and Coulhon T., Analysis and geometry on groups. Cambridge University Press, 1992. MR 95f:43008

Department of Mathematics, Malott Hall, Cornell University, Ithaca, New York 14853-4201

E-mail address: bendikov@math.cornell.edu

Department of Mathematics, Malott Hall, Cornell University, Ithaca, New York 14853-4201

E-mail address: lsc@math.cornell.edu 